\section{A phase I/II study of the combination of quizartinib with azacitidine or low-dose cytarabine for the treatment of patients with acute myeloid leukemia or myelodysplastic syndrome}

\author{
Mahesh Swaminathan, ${ }^{1,2}$ Hagop M. Kantarjian, ${ }^{1}$ Mark Levis, ${ }^{3}$ \\ Veronica Guerra, ${ }^{1}$ Gautam Borthakur, ${ }^{1}$ Yesid Alvarado, ${ }^{1}$ Courtney D. DiNardo, ${ }^{1}$ \\ Tapan Kadia, ${ }^{1}$ Guillermo Garcia-Manero, ${ }^{1}$ Maro Ohanian, ${ }^{1}$ Naval Daver, ${ }^{1}$ \\ Marina Konopleva, ${ }^{1}$ Naveen Pemmaraju, ${ }^{1}$ Alessandra Ferrajol, ${ }^{1}$ \\ Michael Andreeff, ${ }^{1}$ Nitin Jain, ${ }^{1}$ Zeev Estrov, ${ }^{1}$ Elias J. Jabbour, ${ }^{1}$ \\ William G. Wierda, ${ }^{1}$ Sherry Pierce, ${ }^{1}$ Maria Rhona Pinsoy, ${ }^{1}$ Lianchun Xiao, ${ }^{4}$ \\ Farhad Ravandi ${ }^{1}$ and Jorge E. Cortes ${ }^{1,5}$ \\ ${ }^{1}$ Department of Leukemia, University of Texas MD Anderson Cancer Center, Houston, TX; \\ ${ }^{2}$ Department of Medicine, Roswell Park Comprehensive Cancer Center, Buffalo, NY; \\ ${ }^{3}$ Department of Hematological Malignancies, Johns Hopkins Sidney Kimmel \\ Comprehensive Cancer Center, Baltimore, MD; ${ }^{4}$ Department of Biostatistics, University of \\ Texas MD Anderson Cancer Center, Houston, TX and ${ }^{5}$ Georgia Cancer Center at Augusta \\ University, Augusta, GA, USA
}

\section{ABSTRACT}

T he FMS-like tyrosine kinase 3-internal tandem duplication (FLT3-ITD) mutation in acute myeloid leukemia (AML) is associated with poor prognosis. We hypothesized that quizartinib, a selective and potent FLT3 inhibitor, with azacitidine (AZA) or low-dose cytarabine (LDAC) might improve the outcomes in patients with FLT3-ITD-mutated AML. In this open-label phase I/II trial, patients of any age receiving first-salvage treatment for FLT3-ITD AML or age $>60$ years with untreated myelodysplastic syndrome or AML were treated with quizartinib plus AZA or LDAC. Seventy-three patients were treated (34 frontline, 39 first salvage). With regard to previously untreated patients, the composite response (CRc) rate was $87 \%$ ( $n=13 / 15: 8$ complete responses [CR], 4 CR with incomplete hematologic recovery [CRi], $1 \mathrm{CR}$ without platelet recovery [CRp]) among the patients treated with quizartinib/AZA and 74\% ( $n=14 / 19: 1 \mathrm{CR}, 8 \mathrm{CRi}$, 5 CRp) among those treated with quizartinib/LDAC. The median overall survival was 19.2 months for the cohort treated with quizartinib/AZA cohort and 8.5 months for the patients treated with quizartinib/LDAC; the corresponding relapse-free survival figures were 10.5 and 6.4 months, respectively. With regard to previously treated patients, the CRc rate was $64 \%(n=16 / 25$ in the quizartinib/AZA cohort and $29 \%(n=4 / 14))$ in the quizartinib/LDAC cohort. The median overall survival for patients treated with quizartinib/AZA and quizartinib/LDAC was 12.8 versus 4 months, respectively. OTc prolongation grade 3 occurred in only one patient in each cohort. Quizartinib-based combinations, particularly with AZA, appear effective in both frontline and first salvage therapy for patients with FLT3-ITD-mutated AML and are well tolerated. ClinicalTrials.gov identifier: NCT01892371.

\section{Introduction}

One of the most common types of genetic alterations in acute myeloid leukemia (AML) are mutations in the FMS-like tyrosine kinase 3 (FLT3) gene, which occurs in approximately $30 \%$ of all newly diagnosed AML cases. ${ }^{1}$ Internal tandem duplications (ITD) are the most frequent FLT3 mutations, occurring in about 20\% to $30 \%$ of patients with AML., ${ }^{2,3}$ FLT3-ITD mutations confer an adverse prognosis in patients
Haematologica 2021

Volume 106(8):2121-2130

\section{Correspondence:}

JORGE CORTES

jorge.cortes@augusta.edu

Received: June 15, 2020.

Accepted: December 11, 2020.

Pre-published: April 15, 2021.

https://doi.org/10.3324/haematol.2020.263392

(C)2021 Ferrata Storti Foundation

Material published in Haematologica is covered by copyright. All rights are reserved to the Ferrata Storti Foundation. Use of published material is allowed under the following terms and conditions:

https://creativecommons.org/licenses/by-nc/4.0/legalcode. Copies of published material are allowed for personal or internal use. Sharing published material for non-commercial purposes is subject to the following conditions:

https://creativecommons. org//icenses/by-nc/4.0/legalcode, sect. 3. Reproducing and sharing published material for commercial purposes is not allowed without permission in writing from the publisher. 
treated with standard chemotherapy. ${ }^{46}$ Several tyrosine kinase inhibitors, such as lestaurtinib, sunitinib, sorafenib, and midostaurin, have been investigated in patients with FLT3-ITD-mutated AML.,7-10 Of them, the Food and Drug Administration (FDA) has approved only midostaurin in combination with standard chemotherapy for the treatment of patients with FLT3-mutated AML. ${ }^{11}$ Next-generation tyrosine kinase inhibitors, such as gilteritinib and quizartinib, used as single agents have greater antileukemic activity because of their complete FLT3 kinase inhibition. ${ }^{2,12}$

Quizartinib is a type II inhibitor that specifically targets the inactive conformation of the FLT3 kinase domain. ${ }^{13}$ This selective affinity makes it active only against FLT3-ITD mutations, whereas type I inhibitors are active against both FLT3-ITD and tyrosine kinase domain mutations. Quizartinib and gilteritinib have been reported to have significant clinical activity in patients with FLT3-mutated refractory/relapsed (R/R) AML. ${ }^{14,15}$ The QuANTUM-R trial showed a significant improvement in survival of patients with FLT3-ITD AML who received first salvage therapy with quizartinib compared to the survival of those given standard chemotherapy. ${ }^{16}$ The FDA recently approved gilteritinib for the treatment of patients with FLT3-mutated R/R AML. ${ }^{17}$ Azacitidine (AZA), a hypomethylating agent, and cytarabine are standard agents for the treatment of AML in patients not eligible for standard chemotherapy. It has been suggested that combinations of AZA with FLT3 inhibitors (sorafenib, midostaurin) produce higher response rates compared to those expected with FLT3 inhibitors given as single agents. ${ }^{18-21}$

Here we describe the results of a phase I/II, open-label, single-institution study that assessed the efficacy and safety of quizartinib plus AZA (quizartinib/AZA) and quizartinib plus low-dose cytarabine (LDAC, quizartinib/LDAC) in previously untreated elderly patients with AML, or patients with R/R AML at first salvage.

\section{Methods}

\section{Study design}

This was a single-institution phase I/II, two-arm, open-label study. The primary objective of the phase I part of this study was to assess the safety and determine the dose-limiting toxicity and maximum-tolerated dose of these combinations. The primary objective of the phase II portion of the study was to determine the efficacy of the combination of quizartinib with either AZA or LDAC in patients with AML or high-risk myelodysplastic syndrome. Secondary objectives of phase I included assessment of the efficacy of the treatment regimens. Secondary objectives of phase II included a determination of the safety of the quizartinib-based combinations. All patients signed an informed consent form approved by the Institutional Review Board. The study was conducted in accordance with the Declaration of Helsinki and registered with the. ClinicalTrials.gov identifier, NCT01892371.

AZA $\left(75 \mathrm{mg} / \mathrm{m}^{2} /\right.$ day) was administered subcutaneously or intravenously once daily, and LDAC (20 mg flat dose) was administered subcutaneously twice daily for the first 7 days and 10 days of every 28-day cycle, respectively. The decision of whether to use AZA or LDAC was based on the treating physician's choice. In both regimens quizartinib was administered orally daily for 28 days of every cycle except for the first cycle, in which it was started on day 5 of the cycle. The first six patients in both the AZA and LDAC cohorts received quizartinib at the target dose of 60 $\mathrm{mg} /$ day to determine tolerability (i.e., run-in phase). According to the study design, if one or no patient experienced dose-limiting toxicity at this dose, this would be used as the recommended phase II dose. There were no attempts to explore higher doses of quizartinib. If two or more patients experienced dose-limiting toxicity at this dose, a reduced dose level of $30 \mathrm{mg} /$ day was to be explored. Dose-limiting toxicity, defined as any grade $\geq 3$ nonhematologic toxicity at least possibly related to quizartinib or prolonged myelosuppression for $\geq 6$ weeks without evidence of leukemia, was not identified in any of the first six patients treated with each combination. Thus $60 \mathrm{mg} /$ day was used in all patients in the phase II part of the study. The use of hydroxyurea was allowed during the first cycle only. Intrathecal chemotherapy was allowed if clinically indicated.

For the phase I part of the study (run-in phase) only, patients with wild-type (WT)-FLT3 were eligible. All patients with FLT3WT were to receive quizartinib/AZA. However, one patient with a history of FLT3-ITD was enrolled in the quizartinib/LDAC cohort, although FLT3-ITD was not detected at the time of enrollment. Only patients with FLT3-ITD were eligible for the phase II part of the study.

\section{Patients}

Patients with myelodysplastic syndrome, or AML (excluding acute promyelocytic leukemia) meeting at least one of the following criteria were eligible: age $\geq 18$ years with $R / R$ disease who had received no more than one prior treatment regimen; or age $\geq 60$ years with previously untreated disease considered not suitable for intensive chemotherapy. Eastern Cooperative Oncology Group Performance Status $\leq 2$ and adequate organ function were required.

Exclusion criteria included other malignancies concurrent or in remission for $<6$ months prior to enrollment, or clinically active central nervous system leukemia. Patients whose QTc interval, calculated using the Fridericia correction factor (QTCF), was $>450$ ms at screening were excluded.

\section{Tolerability and safety assessments}

All patients who received at least one dose of any of the treatment were evaluable for toxicity. Physical examination, complete blood count, and biochemical analyses were performed at baseline and throughout the study. Electrocardiograms were recorded at screening and on days $1,5,8$, and 12 (before treatment on days 1 , 5 , and 8; 2-6 hours after treatment on days 5 and 12). Triplicate electrocardiograms were obtained for the first three cycles.

\section{Response to treatment}

Bone marrow aspirations and/or biopsies were performed between days 21 and 35 of cycle 1, and every one to three cycles thereafter (monthly until remission, then no later than every 3 months). All patients who received at least one dose of therapy were included in the intention-to-treat (ITT) analysis for response to therapy; patients who completed a full course of treatment (AZA for 7 days or LDAC for 10 days, and quizartinib for 24 days in cycle 1) were evaluable for response in the per-protocol analysis. Responses were defined according to the International Working Group 2003 criteria. ${ }^{22}$ The composite response (CRc) rate was determined based on the sum of the complete responses (CR), $\mathrm{CR}$ with incomplete hematologic recovery (CRi), CR without platelet recovery (CRp) and CR with partial hematologic recovery (CRh). Measurable residual disease (MRD) was assessed by multicolor flow cytometry with a sensitivity of $0.01 \%$.

\section{Statistical analyses}

Safety was monitored closely using the method of Thall et al..$^{23}$ 
Categorical variables were tabulated with their frequencies, and continuous variables were summarized with descriptive statistics. The Fisher exact test, Wilcoxon rank-sum test, and logistic regression model were applied to evaluate the association of response and covariates. The log-rank test and Cox proportional hazards models were used to evaluate the association between overall survival or relapse-free survival with covariates. Survival probabilities were calculated by the Kaplan-Meier method. Comparisons of survival endpoints between the two treatment cohorts are descriptive in nature only as the study was not powered to identify statistical significance. The statistical computations were performed using SAS 9.4 (SAS Institute Inc., Cary NC, USA), S-Plus software v8.2 (TIBCO, Palo Alto, CA, USA), and GraphPad Prism 7 (GraphPad Software, Inc., La Jolla, CA, USA).

\section{Results}

\section{Patients' characteristics}

A total of 73 patients were treated (Figure 1); 34 patients were treated frontline and 39 in a first salvage setting. Fifteen patients received quizartinib/AZA, and 19 received quizartinib/LDAC as frontline treatment. The median age was 75 years (range, 64-82 years) and 70 years (range, 5880 years), respectively (Table 1). Most patients in both cohorts had adverse-risk genetics according to the European LeukemiaNet 2017 classification. All patients were assessed for mutations, using an 81-gene panel, before the start of treatment. The most frequent co-existing mutations were DNMT3A (32\%), RUNX1 (29\%), NPM1 (27\%), TET2 (18\%), and TP53 (6\%).

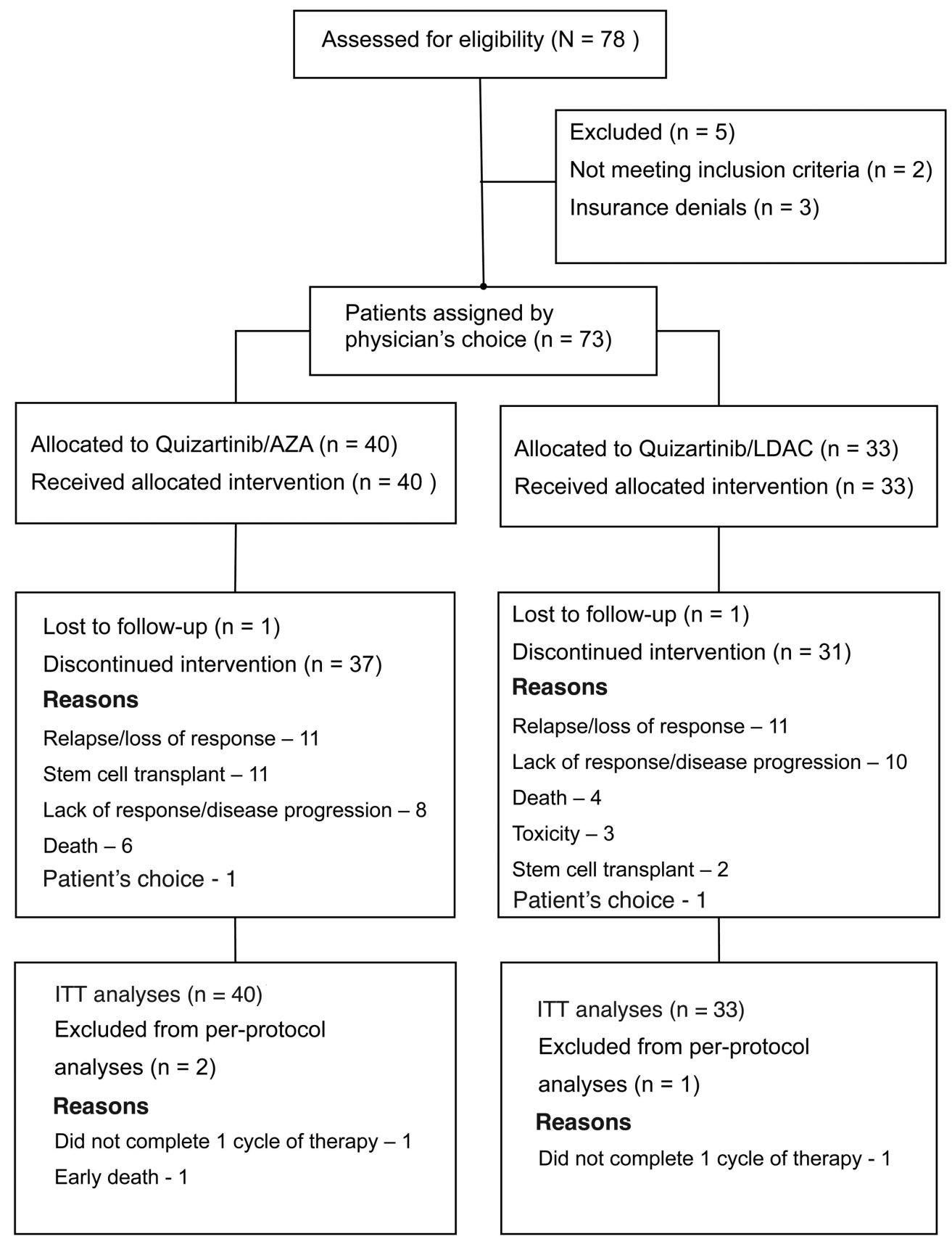

Figure 1. CONSORT flow diagram. AZA: azacitidine; LDAC: low-dose cytarabine; ITT: intention-to-treat. 
Table 1. Baseline characteristics of all study patients $(n=73)$.

\begin{tabular}{|c|c|c|c|c|c|c|}
\hline \multirow[t]{2}{*}{ Patients' characteristics } & \multicolumn{3}{|c|}{ Frontline } & \multicolumn{3}{|c|}{ Relapsed/Refractory } \\
\hline & $\begin{array}{l}\text { Overall } \\
(n=34)\end{array}$ & $\begin{array}{c}\text { Quizartinib/ } \\
\qquad \begin{array}{c}\text { AZA } \\
(n=15)\end{array}\end{array}$ & $\begin{array}{l}\text { Quizartinib/LDAC } \\
\begin{array}{c}(n=19) \\
\text { N }(\%) \text { or }\end{array}\end{array}$ & $\begin{array}{l}\begin{array}{l}\text { Overall } \\
(n=39)\end{array} \\
\text { [range] }\end{array}$ & $\begin{array}{c}\text { Quizartinib/ } \\
\text { AZAA } \\
(n=25)\end{array}$ & $\begin{array}{c}\text { Quizartinib/ } \\
\text { LDAC } \\
(n=14)\end{array}$ \\
\hline Median age, years & $72[58-82]$ & 75 [64-82] & $70[58-80]$ & $65[24-84]$ & $59[24-76]$ & 70 [54-84] \\
\hline Male & $20(59)$ & $9(60)$ & $11(58)$ & $21(54)$ & $13(52)$ & $8(57)$ \\
\hline \multicolumn{7}{|l|}{ ECOG-PS } \\
\hline $0-1$ & $27(79)$ & $13(87)$ & $14(74)$ & $29(74)$ & $20(80)$ & $9(64)$ \\
\hline 2 & $7(21)$ & $2(13)$ & $5(26)$ & $10(26)$ & $5(20)$ & $5(36)$ \\
\hline $\mathrm{AML}^{\mathrm{a}}$ & $33(97)$ & $14(93)$ & $19(100)$ & $39(100)$ & $25(100)$ & $14(100)$ \\
\hline MDS & $1(3)$ & $1(7)$ & 0 & 0 & 0 & 0 \\
\hline \multicolumn{7}{|l|}{ Prior FLT3 inhibitors } \\
\hline Sorafenib & 0 & 0 & 0 & $7(18)$ & $3(12)$ & $4(29)$ \\
\hline Midostaurin & 0 & 0 & 0 & $2(5)$ & 0 & $2^{b}(14)$ \\
\hline Crenolanib & 0 & 0 & 0 & $1(3)$ & 0 & $1(7)$ \\
\hline \multicolumn{7}{|l|}{ ELN risk stratification group ${ }^{c}$} \\
\hline Favorable & $2(6)$ & 0 & $2(11)$ & $2(5)$ & $1(4)$ & $1(7)$ \\
\hline Intermediate & $11(32)$ & $4(27)$ & $7(37)$ & $25(64)$ & $17(68)$ & $8(57)$ \\
\hline Adverse & $21(62)$ & $11(73)$ & $10(53)$ & $12(31)$ & $7(28)$ & $5(36)$ \\
\hline \multicolumn{7}{|l|}{ FLT3 status } \\
\hline FLT3-WT & 0 & 0 & 0 & $3(8)$ & $2(8)$ & $1^{\mathrm{d}}(7)$ \\
\hline FLT3-ITD $^{+}$ & $34(100)$ & $15(100)$ & $19(100)$ & $35(90)$ & $22(88)$ & $13(93)$ \\
\hline Ratio $<0.5$ & $12(35)$ & $5(33)$ & $7(37)$ & $16(41)$ & $10(40)$ & $6(43)$ \\
\hline$\geq 0.5$ & $22(65)$ & $10(67)$ & $12(63)$ & $19(49)$ & $12(48)$ & $7(50)$ \\
\hline Median FLT3-ITD allelic ratio & $0.71[0.01-5.4]$ & $0.86[0.02-5.4]$ & $0.62[0.01-2.4]$ & $0.69[0.01-3.7]$ & $0.72[0.01-3.7]$ & $0.69[0.07-1.2]$ \\
\hline FLT3-TKD ${ }^{+}$only & & & & $1(3)$ & $1(4)$ & 0 \\
\hline Both FLT3-ITD ${ }^{+} \&$ TKD $^{+}$ & $2(6)$ & $1(7)$ & $1(5)$ & $3(8)$ & $1(4)$ & $2(14)$ \\
\hline \multicolumn{7}{|l|}{ Other frequent mutations ${ }^{\mathrm{e}}$} \\
\hline DNMT3A & $11(32)$ & $7(47)$ & $4(21)$ & $12(36)$ & $9(43)$ & $3(25)$ \\
\hline RUNXI & $10(29)$ & $5(33)$ & $5(26)$ & $4(12)$ & $1(4)$ & $3(25)$ \\
\hline NPM1 & $9(27)$ & $2(13)$ & $7(37)$ & $12(36)$ & $10(48)$ & $2(17)$ \\
\hline TET2 & $6(18)$ & $2(13)$ & $4(21)$ & $7(21)$ & $3(14)$ & $4(33)$ \\
\hline TP53 & $2(6)$ & 0 & $2(11)$ & $2(6)$ & $1(4)$ & $1(7)$ \\
\hline \multicolumn{7}{|l|}{ Laboratory values, median } \\
\hline Bone marrow blasts, $\%$ & 56 [15-97] & 56 [15-84] & $60^{\mathrm{f}}[17-97]$ & $65[6-98]$ & $70[6-98]$ & $49[21-86]$ \\
\hline $\mathrm{WBC}, 10^{9} / \mathrm{L}$ & $6.1[1.1-42]$ & 8 [1.2-42] & $3.3[1.1-21]$ & $5.3[0.5-61]$ & $5.2[0.5-53.4]$ & $5.4[0.9-61]$ \\
\hline Hemoglobin, g/dL & $9.1[7-11.4]$ & $9.1[7.7-10]$ & 9 [7-11.4] & $9.4[7.6-12.8]$ & $9.5[7.6-12.7]$ & $9.2[7.7-12.8]$ \\
\hline Platelets, $10^{9} / \mathrm{L}$ & 34 [7-377] & 54 [14-377] & $31[7-75]$ & $25[4-454]$ & $22[4-99]$ & 25 [12-454] \\
\hline
\end{tabular}

AZA: azacitidine; LDAC: low-dose cytarabine; ECOG-PS: Eastern Cooperative Oncology Group-Performance Status; AML: acute myeloid leukemia; MDS: myelodysplastic syndromes; ELN: European LeukemiaNet; ITD: internal tandem duplication;TKD: tyrosine kinase domain;WBC: white blood cells. ${ }^{\text {SSix }}$ patients in the AZA and 12 in the LDAC cohort treated frontline had secondary AML. Three patients each in both the AZA and LDAC cohorts given with first salvage therapy had secondary AML. ${ }^{\mathrm{b}}$ One patient who received LDAC was treated with both sorafenib and midostaurin prior to quizartinib. ${ }^{c}$ Classified according to the European LeukemiaNet 2017 . ${ }^{\mathrm{d}}$ One patient had a prior FLT3-TKD mutation that was negative at the time of starting treatment. ${ }^{\text {The }}$ 81-gene panel was not used for mutational analysis prior to starting treatment in four patients given first salvage treatment with AZA and two given LDAC. 'Two patients were diagnosed based on circulating blast percentages of $22 \%$ and $25 \%$.

Of the 39 patients with R/R AML, 25 patients (64\%) were treated with quizartinib/AZA and $14(36 \%)$ with quizartinib/LDAC. Their median age was 59 years (range, 24-76 years) and 70 years (range, 54-84 years), respectively. Prior to receiving quizartinib, three $(12 \%)$ patients in the quizartinib/AZA cohort and seven $(50 \%)$ in the quizartinib/LDAC cohort had received other FLT3 inhibitors, including sorafenib $(n=7)$, crenolanib $(n=2)$, and midostaurin $(n=1)$. Similarly, five $(13 \%)$ patients had received prior therapy with AZA for AML and four (10\%) for the treatment of a prior myelodysplastic syndrome.
Mutation data (from the 81-gene panel) were not available for four patients in the quizartinib/AZA cohort and two in the quizartinib/LDAC cohort. Among the 33 patients with available molecular information, the most common coexisting mutations were NPM1 (36\%), DNMT3A (36\%), TET2 $(21 \%)$, WT1 $(15 \%)$, IDH2 (15\%), IDH1 (12\%), and TP53 (6\%).

\section{Response to therapy}

All 73 patients received at least one dose of study treatment and were included in the ITT analysis. Among them, 
Table 2. Summary of responses in the intention-to-treat population $(n=73)$.

\begin{tabular}{|c|c|c|c|c|c|c|}
\hline Responses & $\begin{array}{l}\text { Overall } \\
(n=34)\end{array}$ & $\begin{array}{c}\text { Frontline } \\
\text { Quizartinito/ } \\
\text { AZA } \\
(n=1.5)\end{array}$ & $\begin{array}{c}\text { Quizartinib/ } \\
\text { LDAC } \\
(n=19)\end{array}$ & $\begin{array}{l}\text { Overall } \\
(n=39)\end{array}$ & $\begin{array}{c}\text { apsed/Refrac } \\
\text { Quizartinilo/ } \\
\text { AZA } \\
(n=25)\end{array}$ & $\begin{array}{c}\text { Quizartinib/ } \\
\text { LDAC } \\
(n=14)\end{array}$ \\
\hline $\mathrm{CR}$ & $9(26)$ & $8(53)$ & $1(5)$ & $3(8)$ & $2(8)$ & $1(7)$ \\
\hline CRi & $12(35)$ & $4(27)$ & $8(42)$ & $14(36)$ & $12(48)$ & $2(14)$ \\
\hline CRp & $6(18)$ & $1(7)$ & $5(26)$ & $3(8)$ & $2(8)$ & $1(7)$ \\
\hline CRc & $27(79)$ & $13(87)$ & $14(74)$ & $20(51)$ & $16(64)$ & $4(29)$ \\
\hline$P R$ & 0 & 0 & 0 & $2(5)$ & 0 & $2(14)$ \\
\hline ORR & $27(79)$ & $13(87)$ & $14(74)$ & $22(56)$ & $16(64)$ & $6(43)$ \\
\hline NR & $5(15)$ & $1(7)$ & $4(21)$ & $16(41)$ & $8(32)$ & $8(57)$ \\
\hline
\end{tabular}

AZA: azacitidine; LDAC: low-dose cytarabine; CR: complete response; CRi: CR with incomplete hematologic recovery; CRp: CR without platelet recovery; CRh: CR with partial hematologic recovery; $\mathrm{CRc}$ : composite response rate (CR+CRi+CRp); PR: partial response; ORR: overall response rate (CRc+PR); NR:, no response.

Table 3. Summary of responses in evaluable patients* $(n=70)$.

\begin{tabular}{|c|c|c|c|c|}
\hline \multirow{3}{*}{ Responses } & \multicolumn{2}{|c|}{ Frontline } & \multicolumn{2}{|c|}{ Relapsed/Refractory } \\
\hline & $\begin{array}{c}\text { Quizartinilo/AZA } \\
(n=14)\end{array}$ & $\begin{array}{c}\text { Quizartinib/LDAC } \\
(n=18)\end{array}$ & $\begin{array}{c}\text { Quizartinilb/AZA } \\
(\mathrm{n}=24)\end{array}$ & $\begin{array}{c}\text { Quizartinib/LDAC } \\
\qquad(n=14)\end{array}$ \\
\hline & \multicolumn{4}{|c|}{ N $(\%)$ or [range] } \\
\hline $\mathrm{CR}$ & $8(57)$ & $1(6)$ & $2(8)$ & $1(7)$ \\
\hline CRi & $4(29)$ & $8(44)$ & $12(50)$ & $2(14)$ \\
\hline CRp & $1(7)$ & $5(28)$ & $2(8)$ & $1(7)$ \\
\hline $\mathrm{CRh}$ & 0 & 0 & 0 & 0 \\
\hline CRc & $13(93)$ & $14(78)$ & $16(67)$ & $4(29)$ \\
\hline PR & 0 & 0 & 0 & $2(14)$ \\
\hline ORR & $13(93)$ & $14(78)$ & $16(67)$ & $6(43)$ \\
\hline NR & $1(7)$ & $4(22)$ & $8(33)$ & $8(57)$ \\
\hline Time to best response, months & $3.2[0.9-6.3]$ & $1.5[0.9-3]$ & $1[0.9-4.8]$ & $1[0.9-8.7]$ \\
\hline Duration of response ${ }^{b}$, months & $4.2[0.2-30]$ & $3.7[0.2-17]$ & $2.5[0.1-19.4]$ & $2.4[0.5-7.2]$ \\
\hline \multicolumn{5}{|l|}{$\mathrm{MRD}^{\mathrm{c}}$} \\
\hline MRD assessed in responders & $11(85)$ & $12(86)$ & $13(81)$ & $3(50)$ \\
\hline MRD negative $^{\mathrm{d}}$ & $5(45)$ & $3(25)$ & $6(46)$ & $2(67)$ \\
\hline CR & $4(80)$ & $1(33)$ & $1(17)$ & $1(50)$ \\
\hline CRi & $1(20)$ & $1(33)$ & $5(83)$ & $1(50)$ \\
\hline CRp & 0 & $1(33)$ & 0 & 0 \\
\hline Time to MRD negativity prior to SCT, months & $4.7[1-5.9]$ & $0.9[0.9-3]$ & $2[0.9-4.8]$ & $1.4[0.9-1.9]$ \\
\hline \multicolumn{5}{|c|}{$\begin{array}{l}\text { AZA: azacitidine; LDAC: low-dose cytarabine; CR: complete response; CRi: CR with incomplete hematologic recovery; CRp: CR without platelet recovery; CRh: CR with partial } \\
\text { hematologic recovery; CRc: composite response rate (CR+CRi+CRp+CRh); PR: partial response; ORR: overall response rate (CRc+PR); NR:, no response; MRD: minimal residual } \\
\text { disease; SCT: stem cell transplantation. }{ }^{\circ} \text { Only patients who completed at least one course of study treatment were included in the per-protocol response assessment (1 patient } \\
\text { in the AZA cohort died early and } 1 \text { in each cohort did not complete a course of study treatment due to SCT in } 1 \text { case and atrial fibrillation in the other). }{ }^{\circ} \text { Patients proceeded } \\
\text { to SCT were censored at the date of SCT. One patient treated frontline in quizartinib/LDAC and as first salvage in quizartinib/AZA cohort has an ongoing response. }{ }^{.} \mathrm{MRD} \text { was } \\
\text { assessed by multicolor flow cytometry. }{ }^{\mathrm{C} C e n s o r e d ~ p a t i e n t s ~ w h o ~ a c h i e v e d ~ M R D ~ n e g a t i v i t y ~ a f t e r ~ S C T ~ i n ~ t h e ~ g r o u p ~ t r e a t e d ~ f r o n t l i n e ~(~}=4 ; \text {; AZA - } 3 \text {, LDAC -1) and in those with } \\
\text { relapsed/refractory disease (AZA - 3). }\end{array}$} \\
\hline
\end{tabular}

70 (38 in the quizartinib/AZA cohort, 32 in the quizartinib/LDAC cohort) completed at least one cycle of study treatment and were included for per-protocol response assessment. Three patients were not evaluable for perprotocol response: one proceeded to a stem-cell transplant (SCT) after 14 days of treatment, one developed atrial fibrillation on day 4 (prior to receiving quizartinib), and one died early on day 7 of multi-organ failure secondary to sepsis from an unknown organism. Among the ITT population treated frontline, the $\mathrm{CR} c$ rate $(\mathrm{CR}+\mathrm{CRi}+\mathrm{CRp}+$ CRh) was $87 \%$ ( $n=13 / 15$ patients: 8 CR, 4 CRi, 1 CRp) (Table 2) in those treated with quizartinib/AZA and 74\% ( $n=14 / 19$ patients: $1 \mathrm{CR}, 8 \mathrm{CRi}, 5 \mathrm{CRp}$ ) in those treated with quizartinib/LDAC. The per-protocol response data are shown in Table 3 . The median duration of response (time from best response to death/disease progression/date of SCT or last follow-up/off treatment) was 4.2 months (range, 0.2 - 30 months) among patients treated with quizartinib/AZA and 3.7 months (range, 0.2 17 months) among those treated with quizartinib/LDAC. Among responders, MRD was assessed in 11 patients $(85 \%)$ in the quizartinib/AZA cohort and $12(86 \%)$ in the quizartinib/LDAC cohort. MRD was undetectable in five (45\%) patients treated with quizartinib/AZA and three (25\%) with quizartinib/LDAC (excluding patients who achieved MRD-negative status following SCT). Four patients (29\%) treated with quizartinib/AZA and one (6\%) with quizartinib/LDAC proceeded to SCT after achieving CRc. MRD was assessed in all responders before SCT, and only one of them (treated with quizartinib/AZA) was MRD-negative before undergoing the transplant. With regard to the ITT population with $\mathrm{R} / \mathrm{R}$ 
A

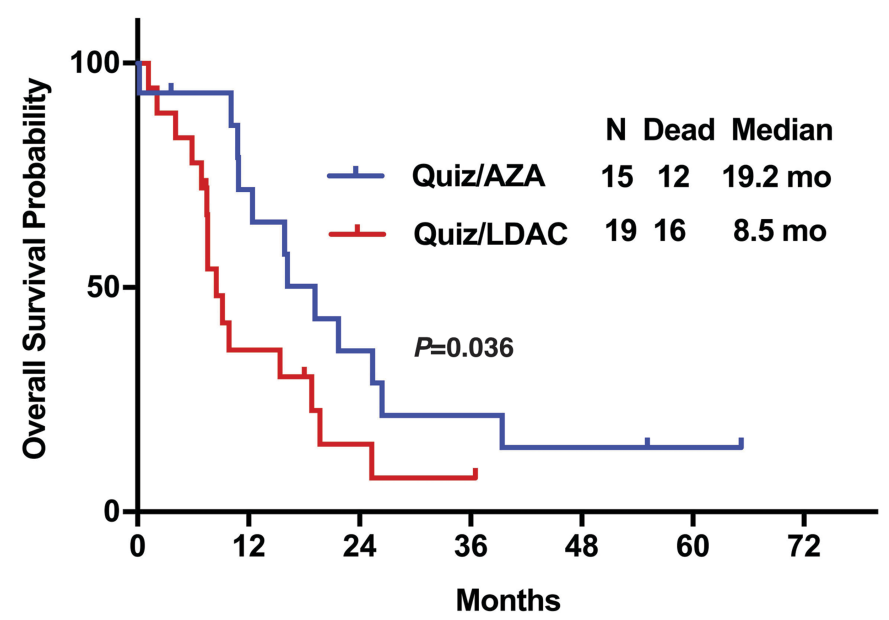

Figure 2. Survival of patients with FLT3-ITD ${ }^{+}$acute myeloid leukemia treated frontline with quizartinib in combination with azacitidine or low-dose cytarabine. (A) Overal survival. (B) Relapse-free survival. Quiz: quizartinib; AZA: azacitidine; LDAC: low-dose cytarabine; mo: months.
B

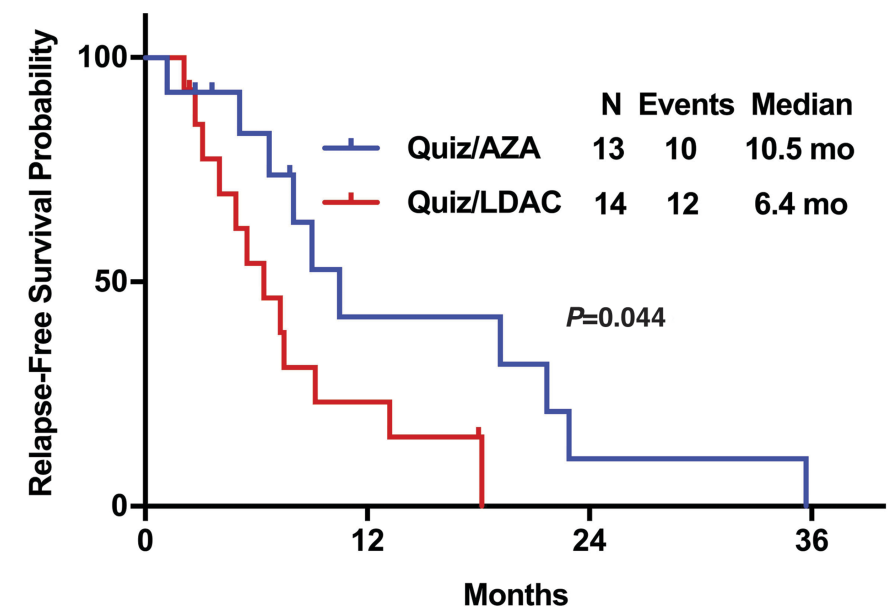

No. at risk

Quiz/AZA 13

Quiz/LDAC 14

5

2

1

4

0
AML, CRc was observed in 64\% ( $n=16$ patients: 2 CR, 12 CRi, 2 CRp) of the quizartinib/AZA cohort and 29\% ( $n=4$ patients: $1 \mathrm{CR}, 2 \mathrm{CRi}, 1 \mathrm{CRp}$ ) in the quizartinib/LDAC cohort (Table 2). The per-protocol response data are shown in Table 3. The median duration of response was 2.5 months (range, 0.1 - 19.4 months) among the patients treated with quizartinib/AZA and 2.4 months (range, 0.5 7.2 months) among those treated with quizartinib/LDAC. Six patients $(25 \%)$ in the quizartinib/AZA cohort and one $(7 \%)$ in the quizartinib/LDAC cohort received SCT after achieving CRc. Among the three patients previously exposed to FLT3 inhibitors and treated with quizartinib/AZA, two achieved a CRi and one a CRp (prior sorafenib). Similarly, two of seven (29\%) such patients treated with quizartinib/LDAC had responses: one achieved a CRi and one had a PR (prior sorafenib). One of the four patients with prior exposure to AZA for AML achieved a CR with quizartinib/AZA.

\section{Survival}

At a median follow-up of 55.1 months (range, 0.2 - 65.2 months), $12(16 \%)$ patients remain alive (8 [20\%] in the
quizartinib/AZA cohort and 4 [12\%] in the quizartinib/LDAC cohort). The median overall survival for all patients treated frontline was 12.4 months. With the caveat that the study was not powered to determine a survival benefit, the median overall survival was longer in patients treated frontline with quizartinib/AZA than in those treated with quizartinib/LDAC (19.2 vs. 8.5 months, respectively; $P=0.036$ ) (Figure 2 ). The median relapse-free survival for all patients treated frontline in this trial was 8 months: it was longer for patients treated with quizartinib/AZA compared to those treated with quizartinib/LDAC (10.5 vs. 6.4 months, respectively; $P=0.044$ ) (Figure 2). Patients with R/R AML had a median overall survival of 6.2 months, with a trend to longer overall survival for those treated with quizartinib/AZA (12.8 vs. 4 months, $P=0.053$ ). There was no difference in the median relapse-free survival between the two treatment groups (overall, 5.8 months; 5.8 vs. 6.2 months by treatment arm, respectively; $P=0.804$ ) (Figure 3 ). We did not find any specific factors that significantly influenced CRc or survival in either the frontline or R/R treatment settings in the two cohorts (Online Supplementary Figures S1 and S2, 
Online Supplementary Tables S1-S4). TET2 mutation was found to have a negative impact on CRc, but only 13/73 patients had a TET2 mutation (Online Supplementary Tables $S 5$ and $S 6$ ).

At the last follow-up of all patients, two (5\%) in the quizartinib/AZA cohort and one $(3 \%)$ in the quizartinib/LDAC cohort continued to receive study treatment (Table 4). Disease relapse or loss of response was the most common reason for study discontinuation in both cohorts. Molecular data at the end of treatment were available for 41 patients (including data collected using an 81 -gene panel in 9 patients). New mutations identified at the end of treatment included FLT3-D835 (11/41, 27\%), IDH1 (2/9, 22\%), RAS (2/11, 18\%), and KIT (1/9, 11\%) (Online Supplementary Figure S3). Eleven (28\%) patients in the quizartinib/AZA cohort and two $(6 \%)$ in the quizartinib/LDAC cohort proceeded to SCT. Three patients $(23 \%)$ treated with quizartinib/AZA relapsed after a median time of 3.2 months (range, 2.7 - 18 months) following SCT. Seven patients $(54 \%)$ are alive after SCT without any evidence of disease ( 5 treated with quizartinib/AZA and 2 treated with quizartinib/LDAC). Of the six patients who died after SCT, three died in CR/CRi/CRp from multi-organ failure secondary to Klebsiella pneumoniae bacteremia, late gastrointestinal graft-versus-host disease, and an unknown cause, respectively. The three other patients had relapsed disease and died, one each, from multi-organ failure secondary to enteroviral pneumonia, intracerebral hemorrhage, and renal failure.

\section{Safety and dose reduction}

The most common treatment-emergent adverse events are listed in Table 5. Among grade $\geq 3$ adverse events, febrile neutropenia and pneumonia were frequently reported in both cohorts. Other common grade $\geq 3$ adverse events that occurred in the cohort of patients treated with quizartinib/AZA were hypokalemia (33\%), hypotension (24\%), and hypophosphatemia (18\%). The median time to absolute neutrophil count recovery (neutrophils $>1 \mathrm{x}$ $10^{9} / \mathrm{L}$ ) for all cycles in patients treated frontline was 32 days (range, 8 - 125 days): it was 35 days (range, 9 - 125 days in the quizartinib/AZA cohort and 27 days (range, 8 - 68 days in the quizartinib/LDAC cohort. Similarly, the median time to platelet recovery (platelets $>100 \times 10^{9} / \mathrm{L}$ ) for all cycles was 29 days (range, 7 - 125 days), 30 days (range, 7 - 125 days) in the quizartinib/AZA cohort and 29 days (range, 25 - 60 days) in the quizartinib/LDAC cohort. The median times to recovery of absolute neutrophil count and platelet count were similar in R/R patients.

QTc prolongation was observed infrequently. Only one (3\%) patient had grade 3 , and three $(9 \%)$ patients had grade 1-2 OTc prolongation in the quizartinib/AZA cohort, and one $(3 \%)$ had grade 3 prolongation in the quizartinib/LDAC cohort (Online Supplementary Figures $S_{4}$ and 55$)$. One (3\%) patient who received quizartinib/AZA as frontline treatment died early (day 7 of treatment) due to multi-organ failure secondary to sepsis from an unknown organism.

A total of nine $(23 \%)$ patients required a dose modification in the quizartinib/AZA cohort. Of them, three patients received quizartinib for only 7 days, 14 days, and 16 days in the first cycle due to the development of multiorgan failure, SCT, and febrile neutropenia, respectively. The quizartinib dose was decreased to $30 \mathrm{mg}$ in six patients (4 due to grade 3 myelosuppression, 1 due to
Table 4. Summary of disposition of all study patients $(n=73)$.

\begin{tabular}{|c|c|c|}
\hline Reasons & $\begin{array}{l}\text { tartinitb/ } \\
(n=40)\end{array}$ & $\begin{array}{l}\text { artinilb/LDAC } \\
(n=33)\end{array}$ \\
\hline Relapse/loss of response & $11(28)$ & $11(33)$ \\
\hline Stem cell transplant & $11(28)$ & $2(6)$ \\
\hline Lack of response/disease progression & $8(20)$ & $10(30)$ \\
\hline Death $^{\mathrm{a}}$ & $6(15)$ & $4(12)$ \\
\hline Continuing treatment & $2(5)$ & $1(3)$ \\
\hline Patient's choice $^{b}$ & $1(3)$ & $1(3)$ \\
\hline Lost to follow-up & $1(3)$ & $1(3)$ \\
\hline Infection/toxicity ${ }^{c}$ & 0 & $3(9)$ \\
\hline
\end{tabular}

AZA: azacitidine; LDAC: low-dose cytarabine. ${ }^{a}$ Of six patients in the AZA cohort who died, one died early, two died in complete response with incomplete hematologic recovery (CRi),two in complete response (CR) and one without response.In the LDAC cohort, four patients died: one in CR without platelet recovery, one in CRi, one with a partial response and one with no response. ${ }^{\mathrm{b}}$ One patient in the AZA cohort chose to be treated at a different institution and one in the LDAC cohort chose to discontinue treatment for financial reasons. ${ }^{c}$ One patient each discontinued treatment due to atrial fibrillation and meningitis. One additional patient developed atrial fibrillation prior to receiving quizartinib and was taken off study.

grade 2 supraventricular tachycardia, and 1 due to grade 3 QTc prolongation). Similarly, the quizartinib dose was decreased to $30 \mathrm{mg}$ in three patients $(9 \%)$ in the quizartinib/LDAC cohort: in two cases due to grade 3 myelosuppression and in one case due to grade 3 QTc prolongation.

\section{Discussion}

With the limitations of comparisons across studies, the $\mathrm{CRc}$ rates and the median overall survival observed in patients treated frontline with quizartinib/AZA in this study seem to compare favorably to what has been reported for similar combinations using other FLT3 inhibitors. With midostaurin plus AZA, the reported CRc and median overall survival were $31 \%$ and 9 months, respectively. Similarly, with sorafenib plus AZA, the CRc and median overall survival were $70 \%$ and 8.3 months, respectively. ${ }^{21}$ The results with quizartinib/AZA also appear comparable to the results reported with a hypomethylating agent/venetoclax ${ }^{24}$ (CR/Cri: $72 \%$ in FLT3-mutated cases; median overall survival not reached). Although patients with FLT3 mutations had good responses to hypomethylating agents/venetoclax, mutations in FLT3ITD constitute one of the mechanisms of acquired resistance to venetoclax in preclinical models, ${ }^{25,26}$ and have been reported to emerge in some patients as they develop resistance to therapy with venetoclax. ${ }^{27}$ Ongoing clinical studies are trying to overcome this resistance by adding FLT3 inhibitors to venetoclax (gilteritinib - NCT03625505 and quizartinib - NCT03735875).

Quizartinib, as a single agent, was found to produce a CRc of $48 \%$ in patients with R/R AML, who achieved a median overall survival of 6.2 months. ${ }^{16}$ Similarly, AZA monotherapy in R/R AML produced CR/CRi in $16 \%$ of patients, who had a median overall survival of 6.8 months. ${ }^{28}$ The combination of quizartinib/AZA in our study produced a CRc of $67 \%$ with a median overall survival of 12.8 months in patients with R/R AML. Although no comparisons could be made between different studies, higher response rates and longer survival observed with the combination could perhaps be attributed to a possible synergy 
A

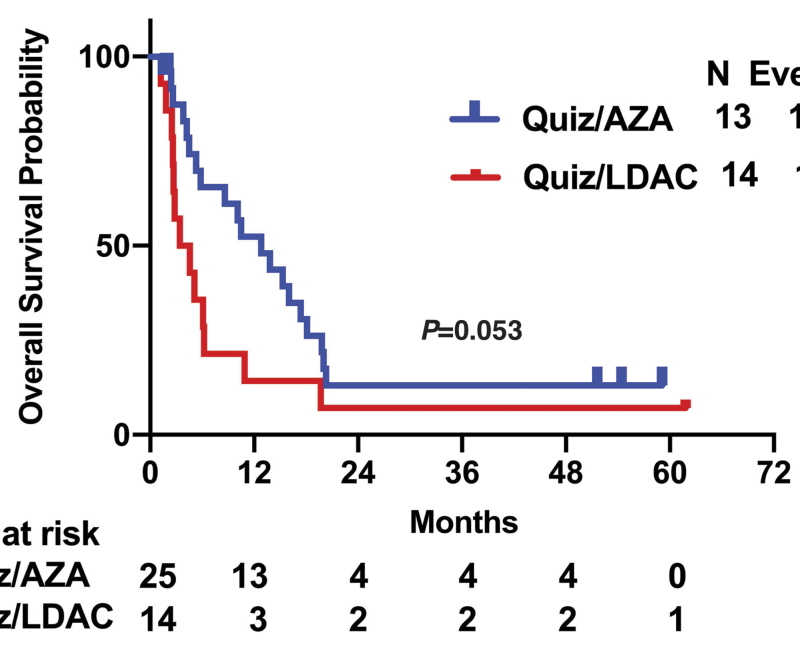

Figure 3. Survival of patients with FLT3ITD $^{+}$relapsed/refractory acute myeloid leukemia given first salvage treatment with quizartinib in combination with azacitidine or low-dose cytarabine. (A) Overall survival. (B) Relapse-free survival. Quiz: quizartinib; AZA: azacitidine; LDAC: low-dose cytarabine; mo: months.

B

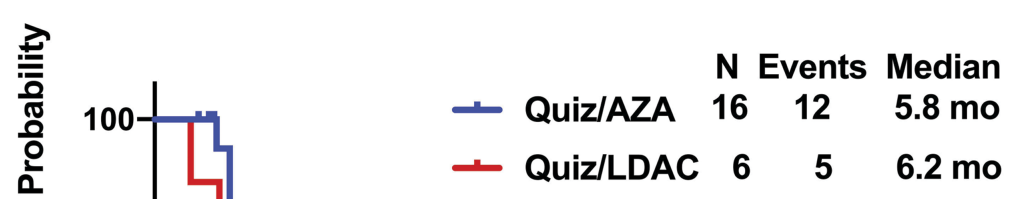

\section{No. at risk \\ Quiz/AZA 16 \\ Quiz/LDAC 6}

between quizartinib and AZA. Similarly, in phase II studies, quizartinib monotherapy showed some activity in patients with FLT3-WT AML with a CRc rate of $32 \% .{ }^{29}$ Our study included only three patients with FLT3-WT, who received quizartinib-based combinations as first salvage treatment but had no response. Because of the paucity of patients, no conclusion could be made on the clinical activity of quizartinib in combination with AZA or LDAC in patients with FLT3-WT AML. However, based on the reported CRc rate of $32 \%$ with quizartinib monotherapy, ${ }_{1}^{14}$ this approach deserves further investigation in this subset of patients.

Prior studies found that point mutations and gatekeeper mutations (F691L) were the common resistant mutations that were acquired following quizartinib therapy. ${ }^{30-32}$ In this series, FLT3-D835 was acquired at the time of progression in $27 \%$ of patients. This is equivalent to what we had reported previously with single-agent FLT3 inhibitors, mostly sorafenib. ${ }^{33}$ Other mutations encountered at the time of progression included EZH2 and CREBBP mutations. However, the mutation analysis was somewhat limited in this study, since mutation data at the time of treatment discontinuation were available for only $41 / 70(59 \%)$ patients (Online Supplementary Figure S3). Similarly, after treatment with gilteritinib, resistance is associated with the emergence of additional mutations, frequently RAS. This may represent a selection of clones, often pre-existing, which emerge as clones with sensitive FLT3 mutations are suppressed by potent FLT3 inhibitors. This suggests that combination therapy would be required to further improve the outcome of patients treated with FLT3 inhibitors. The higher rate of response and more durable responses seen in the present study compared to what has been reported with quizartinib monotherapy are encouraging and merit further investigation.

Like quizartinib monotherapy, quizartinib at a dose of $60 \mathrm{mg}$ in combination with AZA or LDAC was very well tolerated. OTc prolongation was infrequent with only two patients developing grade 3 prolongation and requiring a decrease in quizartinib dose to $30 \mathrm{mg}$. Overall, therapy was well tolerated by our patients in both the cohorts.

As previously reported, a higher FLT3-ITD allelic ratio was found to correlate with the rate of CRc in patients receiving frontline therapy, although the optimal cut point for the ratio was at 0.5 rather than the 0.7 that had been previously proposed. However, no such correlation could be identified for survival. There were no significant correlations between allelic ratio and responses or survival in the salvage cohort (Online Supplementary Figure S6 and 
Table 5. Incidence of common treatment-emergent adverse events in all patients.

\begin{tabular}{|c|c|c|c|c|c|c|}
\hline \multirow[t]{2}{*}{ Adverse events } & \multicolumn{3}{|c|}{$\begin{array}{c}\text { Quizartinib/AZA } \\
\qquad(n=40)\end{array}$} & \multicolumn{3}{|c|}{$\begin{array}{c}\text { Quizartinib/LDAC } \\
(n=33)\end{array}$} \\
\hline & Total & $\begin{array}{c}\text { Grade } 1-2 \\
N(\%)\end{array}$ & Grade 3-5 & Total & $\begin{array}{c}\text { Grade 1-2 } \\
\text { N (\%) }\end{array}$ & Grade 3-5 \\
\hline
\end{tabular}

\section{Non-hematologic toxicities}

\begin{tabular}{|c|c|c|c|c|c|c|}
\hline Hypomagnesemia & $27(68)$ & $26(65)$ & $1(3)$ & $10(30)$ & $10(30)$ & 0 \\
\hline Hyperbilirubinemia & $25(63)$ & $19(48)$ & $6(15)$ & $9(27)$ & $8(24)$ & $1(3)$ \\
\hline Hypokalemia & $24(60)$ & $11(28)$ & $13(33)$ & $9(27)$ & $6(18)$ & $3(9)$ \\
\hline Hypocalcemia & $23(58)$ & $16(40)$ & $7(18)$ & $9(27)$ & $9(27)$ & 0 \\
\hline Increased ALT & $23(58)$ & $19(48)$ & $4(10)$ & $15(45)$ & $13(39)$ & $2(6)$ \\
\hline Hyponatremia & $17(43)$ & $12(30)$ & $5(13)$ & $8(24)$ & $5(15)$ & $3(9)$ \\
\hline Febrile neutropenia & $16(40)$ & 0 & $16(40)$ & $12(36)$ & 0 & $12(36)$ \\
\hline Elevated creatinine & $13(33)$ & $13(33)$ & 0 & $7(21)$ & $7(21)$ & 0 \\
\hline Hypophosphatemia & $12(30)$ & $5(13)$ & $7(18)$ & $3(9)$ & $2(6)$ & $1(3)$ \\
\hline Pneumonia & $11(28)$ & 0 & $11(28)$ & $19(58)$ & $3(9)$ & $16(48)$ \\
\hline Nausea & $10(25)$ & $9(23)$ & $1(3)$ & $14(42)$ & $12(36)$ & $2(6)$ \\
\hline Fatigue & $9(23)$ & $8(20)$ & $1(3)$ & $10(30)$ & $7(21)$ & $3(9)$ \\
\hline Diarrhea & $8(20)$ & $5(13)$ & $3(8)$ & $13(39)$ & $10(30)$ & $3(9)$ \\
\hline Hypoalbuminemia & $8(20)$ & $6(15)$ & $2(5)$ & $7(21)$ & $7(21)$ & 0 \\
\hline Vomiting & $6(15)$ & $6(15)$ & 0 & $10(30)$ & $8(24)$ & $2(6)$ \\
\hline Rash & $6(15)$ & $4(10)$ & $2(5)$ & $9(27)$ & $8(24)$ & $1(3)$ \\
\hline Hyperkalemia & $5(13)$ & $3(8)$ & $2(5)$ & $4(12)$ & $4(12)$ & 0 \\
\hline Lower limb edema & $5(13)$ & $5(13)$ & 0 & $4(12)$ & $4(12)$ & 0 \\
\hline Generalized weakness & $5(13)$ & $4(10)$ & $1(3)$ & $5(15)$ & $3(9)$ & $2(6)$ \\
\hline Oral mucositis & $5(13)$ & $4(10)$ & $1(3)$ & $8(24)$ & $7(21)$ & $1(3)$ \\
\hline Anorexia & $4(10)$ & $3(8)$ & $1(3)$ & $5(15)$ & $5(15)$ & 0 \\
\hline Constipation & $4(10)$ & $4(10)$ & 0 & $5(15)$ & $5(15)$ & 0 \\
\hline Dizziness & $4(10)$ & $4(10)$ & 0 & $6(18)$ & $5(15)$ & $1(3)$ \\
\hline Abdominal pain & $4(10)$ & $1(3)$ & $3(8)$ & $8(24)$ & $7(21)$ & $1(3)$ \\
\hline Hypotension & $4(10)$ & $1(3)$ & $3(8)$ & $12(36)$ & $4(12)$ & $8(24)$ \\
\hline Atrial fibrillation & $2(5)$ & 0 & $2(5)$ & $3(9)$ & $2(6)$ & $1(3)$ \\
\hline Headache & $1(3)$ & $1(3)$ & 0 & $6(18)$ & $3(9)$ & $3(9)$ \\
\hline Pleural effusion & $1(3)$ & $1(3)$ & 0 & $5(15)$ & $4(12)$ & $1(3)$ \\
\hline Back pain & $1(3)$ & $1(3)$ & 0 & $10(30)$ & $9(27)$ & $1(3)$ \\
\hline QTc prolongation & $1(3)$ & 0 & $1(3)$ & $4(12)$ & $3(9)$ & $1(3)$ \\
\hline Sinus tachycardia & $1(3)$ & 0 & $1(3)$ & $2(6)$ & $2(6)$ & 0 \\
\hline Sinus bradycardia & 0 & 0 & 0 & $3(9)$ & $3(9)$ & 0 \\
\hline \multicolumn{7}{|l|}{ Hematologic toxicities } \\
\hline Thrombocytopenia & $6(15)$ & $1(3)$ & $5(13)$ & $10(30)$ & 0 & $10(30)$ \\
\hline Leukopenia & $3(8)$ & $1(3)$ & $2(5)$ & $14(42)$ & 0 & $14(42)$ \\
\hline Neutropenia & $3(8)$ & $1(3)$ & $2(5)$ & $14(42)$ & 0 & $14(42)$ \\
\hline Anemia & $1(3)$ & $1(3)$ & 0 & $5(15)$ & 0 & $5(15)$ \\
\hline Leukocytosis & 0 & 0 & 0 & $2(6)$ & 0 & $2(6)$ \\
\hline
\end{tabular}

AZA: azacitidine; LDAC, low-dose cytarabine; ALT: alanine transaminase.

Online Supplementary Table S5). Recently, Levis and his colleagues showed that among patients with FLT3-mutated $\mathrm{R} / \mathrm{R}$ AML treated with gilteritinib monotherapy, patients with NPM1 co-mutation had significantly better survival outcomes. ${ }^{34}$ Similarly, NPM1 co-mutation was associated with non-significantly better CRc rates and longer overall survival in our R/R patients (Online Supplementary Figure S2 and Online Supplementary Table S4). In general, these associations follow the same direction as those found in our series, but the small number of patients preclude firm conclusions. These possible interactions remain to be confirmed in larger series.

In conclusion, our study demonstrated that quizartinib, in combination with AZA or LDAC, can be administered safely to patients with FLT3-mutated AML, whether as initial therapy or as first salvage treatment. Our preliminary observations suggest that the combination therapy, particularly with AZA, might improve the probability of response and the response duration in these patients. Prospective randomized studies are warranted to validate our results.

\section{Disclosures}

No conflicts of interest to disclose.

\section{Contributions}

$J C$ and $H K$ designed the study; MS, JC, VG, SP, MRP and $L X$ collected and analyzed the data, and performed the statistical analysis; $M S$ and JC wrote the manuscript. All the authors reviewed the manuscript and approved the version for submission.

\section{Funding}

The study was supported in part by a Cancer Center Support Grant (NCI grant P30 CA016672). 


\section{References}

1. Gilliland DG, Griffin JD. The roles of FLT3 in hematopoiesis and leukemia. Blood. 2002;100(5):1532-1542.

2. Daver N, Schlenk RF, Russell NH, Levis MJ. Targeting FLT3 mutations in AML: review of current knowledge and evidence. Leukemia. 2019;33(2):299-312.

3. Nagel G, Weber D, Fromm E, et al. Epidemiological, genetic, and clinical characterization by age of newly diagnosed acute myeloid leukemia based on an academic population-based registry study (AMLSG BiO). Ann Hematol. 2017; 96(12):1993-2003

4. Frohling S, Schlenk RF, Breitruck J, et al. Prognostic significance of activating FLT3 mutations in younger adults (16 to 60 years) with acute myeloid leukemia and normal cytogenetics: a study of the AML Study Group Ulm. Blood. 2002;100(13): 4372-4380.

5. Kottaridis PD, Gale RE, Frew ME, et al. The presence of a FLT3 internal tandem duplication in patients with acute myeloid leukemia (AML) adds important prognostic information to cytogenetic risk group and response to the first cycle of chemotherapy: analysis of 854 patients from the United Kingdom Medical Research Council AML 10 and 12 trials. Blood. 2001;98(6):1752-1759.

6. Schnittger S, Schoch C, Dugas M, et al. Analysis of FLT3 length mutations in 1003 patients with acute myeloid leukemia: correlation to cytogenetics, FAB subtype, and prognosis in the AMLCG study and usefulness as a marker for the detection of minimal residual disease. Blood. 2002;100(1):59-66

7. Fiedler W, Kayser S, Kebenko M, et al. A phase I/II study of sunitinib and intensive chemotherapy in patients over 60 years of age with acute myeloid leukaemia and activating FLT3 mutations. Br J Haematol. 2015;169(5):694-700.

8. Pratz KW, Cortes J, Roboz GJ, et al. A pharmacodynamic study of the FLT3 inhibitor KW-2449 yields insight into the basis for clinical response. Blood. 2009;113(17): 3938-3946.

9. Smith BD, Levis M, Beran M, et al. Singleagent CEP-701, a novel FLT3 inhibitor, shows biologic and clinical activity in patients with relapsed or refractory acute myeloid leukemia. Blood. 2004; 103(10): 3669-3676.

10. Stone RM, DeAngelo DJ, Klimek V, et al. Patients with acute myeloid leukemia and an activating mutation in FLT3 respond to a small-molecule FLT3 tyrosine kinase inhibitor, PKC412. Blood. 2005;105(1):54-60.
11. Stone RM, Mandrekar SJ, Sanford BL, et al. Midostaurin plus chemotherapy for acute myeloid leukemia with a FLT3 mutation. N Engl J Med. 2017;377(5):454-464.

12. Wander SA, Levis MJ, Fathi AT. The evolving role of FLT3 inhibitors in acute myeloid leukemia: quizartinib and beyond. Ther Adv Hematol. 2014;5(3):65-77.

13. Daver N, Cortes J, Ravandi F, et al. Secondary mutations as mediators of resistance to targeted therapy in leukemia. Blood. 2015;125(21):3236-3245.

14. Cortes J, Perl AE, Dohner $\mathrm{H}$, et al. Quizartinib, an FLT3 inhibitor, as monotherapy in patients with relapsed or refractory acute myeloid leukaemia: an open-label, multicentre, single-arm, phase 2 trial. Lancet Oncol. 2018;19(7):889-903.

15. Perl AE, Altman JK, Cortes J, et al. Selective inhibition of FLT3 by gilteritinib in relapsed or refractory acute myeloid leukaemia: a multicentre, first-in-human, open-label, phase 1-2 study. Lancet Oncol. 2017; 18(8):1061-1075.

16. Cortes JE, Khaled S, Martinelli G, et al. Quizartinib versus salvage chemotherapy in relapsed or refractory FLT3-ITD acute myeloid leukaemia (QuANTUM-R): a multicentre, randomised, controlled, openlabel, phase 3 trial. Lancet Oncol. 2019; 20(7):984-997.

17.Perl AE, Martinelli G, Cortes JE, et al. Gilteritinib or chemotherapy for relapsed or refractory FLT3-mutated AML. N Engl Med. 2019;381(18):1728-1740.

18. Ravandi F, Alattar ML, Grunwald MR, et al. Phase 2 study of azacytidine plus sorafenib in patients with acute myeloid leukemia and FLT-3 internal tandem duplication mutation. Blood. 2013;121(23):4655-4662.

19. Strati P, Kantariian H, Ravandi F, et al. Phase I/II trial of the combination of midostaurin (PKC412) and 5-azacytidine for patients with acute myeloid leukemia an myelodysplastic syndrome. Am J Hematol. 2015;90(4):276-281

20. Gallogly MM, Tomlinson BK, Bunner P, et al. A phase II study of midostaurin and 5azacitidine for elderly patients with acute myeloid leukemia. Blood. 2017;130(Supp 1):1332.

21. Ohanian M, Garcia-Manero G, Levis M, et al. Sorafenib combined with 5-azacytidine in older patients with untreated FLT3-ITD mutated acute myeloid leukemia. Am J Hematol. 2018:93(9):1136-1141.

22. Cheson BD, Bennett JM, Kopecky KJ, et al. Revised recommendations of the International Working Group for Diagnosis, Standardization of Response Criteria, Treatment Outcomes, and Reporting Standards for Therapeutic Trials in Acute Myeloid Leukemia. J Clin Oncol.
2003;21(24):4642-4649

23. Thall PF, Simon RM, Estey EH. Bayesian sequential monitoring designs for singlearm clinical trials with multiple outcomes. Stat Med. 1995;14(4):357-379.

24. DiNardo CD, Pratz K, Pullarkat V, et al Venetoclax combined with decitabine or azacitidine in treatment-naive, elderly patients with acute myeloid leukemia. Blood. 2019;133(1):7-17.

25. Mali RS, Lasater EA, Doyle K, et al. FLT3ITD activation mediates resistance to the BCL-2 selective antagonist, venetoclax, in FLT3-ITD mutant AML models. Blood. 2017;130(Suppl 1):1348.

26. Zhang Q, Pan R, Han L, et al. Mechanisms of acquired resistance to venetoclax in preclinical AML models. Blood. 2015 126(23):328-328.

27. Chyla B, Daver N, Doyle K, et al. Genetic biomarkers of sensitivity and resistance to venetoclax monotherapy in patients with relapsed acute myeloid leukemia. Am Hematol. 2018;93(8):E202-E205.

28. Stahl M, DeVeaux M, Montesinos P, et al. Hypomethylating agents in relapsed and refractory AML: outcomes and their predictors in a large international patient cohort Blood Adv. 2018;2(8):923-932.

29. Cortes JE, Perl AE, Dombret H, et al. Final results of a phase 2 open-label, monotherapy efficacy and safety study of quizartinib (AC220) in patients $\geq 60$ years of age with FLT3 ITD positive or negative relapsed/refractory acute myeloid leukemia. Blood. 2012;120(21):48-48.

30. Albers C, Leischner $H$, Verbeek $M$, et al. The secondary FLT3-ITD F691L mutation induces resistance to AC220 in FLT3-ITD+ AML but retains in vitro sensitivity to PKC412 and sunitinib. Leukemia. 2013, 27(6):1416-1418.

31. Smith CC, Lin K, Stecula A, Sali A, Shah NP. FLT3 D835 mutations confer differential resistance to type II FLT3 inhibitors. Leukemia. 2015;29(12):2390-2392.

32. Smith CC, Paguirigan A, Jeschke GR, et al. Heterogeneous resistance to quizartinib in acute myeloid leukemia revealed by singlecell analysis. Blood. 2017;130(1):48-58.

33. Alvarado Y, Kantarjian HM, Luthra R, et al. Treatment with FLT3 inhibitor in patients with FLT3-mutated acute myeloid leukemia is associated with development of secondary FLT3-tyrosine kinase domain mutations. Cancer. 2014;120(14):21422149

34. Levis MJ, Perl AE, Martinelli G, et al. Effect of gilteritinib on survival in patients with FLT3-mutated (FLT3mut+) relapsed/refractory (R/R) AML who have common AML co-mutations or a high FLT3-ITD allelic ratio. J Clin Oncol. 2019;37(15_suppl):7000. 